\title{
Traitement des polymères par laser à excimères
}

\author{
S. Lazare, M. Bolle, D. Drilhole et S. Mihailov
}

Laboratoire de Pho*ophysique et de Photochimie Moléculaire, URA 348 du CNRS, Université de Bordeaux I, 351 cours de la Libération, 33405 Talence, France

\begin{abstract}
Résumé - Nous faisons le point sur l'évolution de la photoablation obtenue par le rayonnement du laser à excimère en mettant l'accent sur le développement récent des études concernant la formation des structures périodiques submicroniques sur des surfaces de polymères, par faisceau polarisé. Ces modifications de surface sont obtenues par irradiation avec un grand nombre d'impulsions d'énergie légèrement inférieure au seuil d'ablation. Les rides formées ont une période submicronique inférieure à la longueur d'onde du rayonnement utilisée, qui dépend de paramètres comme l'incidence, la longueur d'onde et le polymère utilisé. La vitesse d'apparition de ces structures est étudiée pour évaluer précisément les mécanismes photochimiques mises en jeu. Une période d'induction a été mise en évidence, pendant laquelle la surface reste apparemment lisse. On observe ensuite une phase de croissance rapide des structures, suivie d'une période de maturation et d'une décroissance. Dans certains cas la décroissance s'effectue par la transformation des lignes en points. Ce dernier comportement n'est prévu par aucune des théories existantes. Cette méthode de structuration submicronique des surfaces s'avère importante pour un certain nombre d'applications.
\end{abstract}

\section{Introduction}

La photoablation $\left({ }^{1}\right)$ par laser à excimère permet la gravure directe, microscopique et haute résolution de nombreux matériaux (métaux, céramiques, matériaux durs, mous, inhomogènes). Les matériaux polymères, en général, bénéficient de seuils d'ablation faibles $\left(10\right.$ à $100 \mathrm{~mJ} / \mathrm{cm}^{2}$ ) et de vitesses importantes (de l'ordre du micron par impulsion). Ces propriétés en font une classe de matériaux relativement faciles à travailler, d'où les importantes applications qui en découlent dans les domaines de la gravure, du perçage et de l'usinage des matériaux.

Au cours de ces études, les expérimentateurs se sont souvent intéressés aux modifications survenant au niveau de la surface de la cible. Ces modifications sont très superficielles (ordre de grandeur du micron) puisqu'elles sont limitées par la profondeur de pénétration du rayonnement (donnée par l'inverse du coefficient d'absorption de la Figure 1). En particulier, on peut observer dans certains cas l'apparition d'une 
structuration périodique, dite cohérente, car reliée principalement au caractère ondulatoire du rayonnement. Il semble que l'intensité absorbée par le matériau soit modulée spatialement par une radiation de surface résultant de l'interaction du faisceau incident avec les composantes de Fourier de la surface.

Dans une nouvelle approche expérimentale où l'énergie des impulsions est choisie légèrement en dessous des seuils d'ablation, nous avons mis récemment en évidence une nouvelle classe de structures périodiques submicroniques $(2,3)$. Des rides espacées de 0,2 à $0,7 \mu \mathrm{m}$ dans la direction du champ électrique sont obtenues par absorption de plusieurs milliers d'impulsions de la radiation $\mathrm{ArF}$ ou $\mathrm{KrF}$. La période est déterminée par des paramètres expérimentaux comme la longueur d'onde, lincidence ou la nature du polymère. La cinétique du phénomène fait apparaître d'abord, une période d'induction pendant laquelle la surface est modifiée sans se structurer, puis une phase de croissance et de maturité des structures. Ensuite, pour des irradiations prolongées, on peut observer une disparition ou une évolution vers des structures plus désorganisées.

Dans de nombreuses applications, une rugosité contrôlée d'amplitude submicronique est désirée. Grâce à la simplicité de notre processus d'irradiation les traitements de surface à grande échelle sont envisageables. Des propriétés de surface comme la friction, l'adhésion, etc.. peuvent s'en trouver grandement améliorées.

\section{La photoablation des polymères et les microfabrications}

La photoablation est la conséquence directe de l'absorption rapide d'une grande densité d'énergie lumineuse du laser. Aussi les matériaux traités doivent-ils présenter une absorption suffisante aux longueurs d'onde usuelles du laser à excimères. Ces absorptions sont présentées à la Figure. 1 pour une palette de polymères que nous avons étudiés et qui sont représentatifs des grands types de polymères couramment utilisées dans les applications de tous les jours. Les détails concernant les structures de ces polymères et leurs caractéristiques d'ablation peuvent être trouvés dans la référence 4 , par exemple $\left({ }^{4}\right)$, qui est une revue où les résultats plus anciens sur la photoablation sont décrits. Sur la Figure. 1 on peut voir que les polymères les plus absorbants sont les polyimides. Ceux-ci trouvent d'importantes applications en micro-électronique comme diélectrique dans la fabrication des circuits multicouches et multichips. Siemens $\left({ }^{5}\right)$ et $\operatorname{IBM}\left({ }^{6}\right)$ ont développé beaucoup la photoablation de ce type de polymère pour le microperçage de ces isolants dans la fabrication industrielle des circuits multicouches. Sur la Fig.1 on trouve un certain nombre de polymères qui sont moyennement absorbants (PET) ou fortement absorbants pour la longueur d'onde la plus courte (PS). Il vient ensuite la classe des polymères faiblement absorbants mais dont l'absorption est suffisante pour engendrer l'ablation propre (par exemple le PMMA). Il existe aussi un nombre de polymères qui ne présentent pas d'absorption pour les longueurs d'onde comprises entre 193 et $351 \mathrm{~nm}$. C'est, par exemple le cas des polymères insaturés comme les polyéthylènes, polypropylène et polytétrafluoroéthylène (TEFLON). Pour ceux-là, il est possible de recourir à la technique du dopage $\left({ }^{7}\right.$ ), qui consiste à dissoudre dans le polymère, une petite molécule, dont le rôle est d'absorber la radiation laser. C'est cette technique qui a 


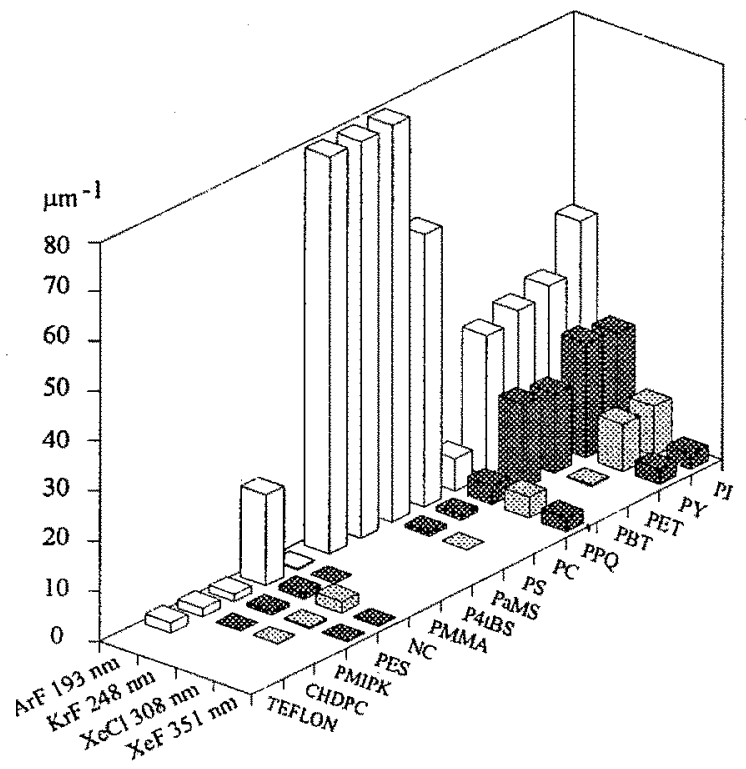

Fig. 1 Coefficients d'absorption (en $\mu \mathrm{m}^{-1}$ ) de polymères typiques pour les longueurs d'onde principales du laser à excimères. Par ordre décroissant d'absorption - PI: polyimide; Py: Pyraline 2550; PET: poly(ethylene terephthalate); $\mathrm{PBT}$ : poly(butylène téréphthalate); PPQ: polyphénylquinoxaline; $\mathrm{PC}$ : polycarbonate; PS: polystyrene; PaMS: poly(alpha-méthyle styrène); P4tBS: poly(4-tertio-butyle styrène); PMMA: poly(métacrylate de méthyle); NC: dinitrocellulose; PES: poly(ethylene succinate); PMIPK: poly(méthyle, isobutyle cétone); CHDPC: polycarbonate du cyclohexanediol; et TEFLON: poly(tetrafluoroethylène).

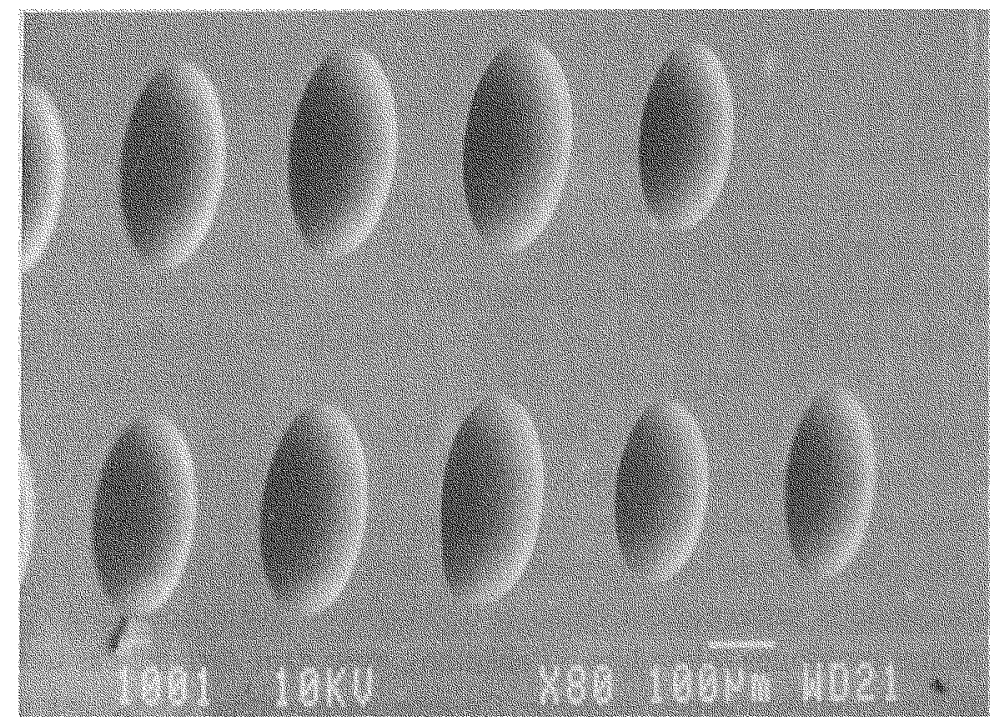

Fig. 2 Exemple de microfabrication obtenue par photoablation du TEFLON AF: matrice de microlentilles de diamètre $250 \mu \mathrm{m}$ et de focale $300 \mu \mathrm{m}$. 
été appliquée dans la fabrication des matrices de microlentilles en TEFLON $\operatorname{AF}\left({ }^{8}\right)$ de la Figure.2. Les matrices de microlentilles deviennent des composants standard dans les technologies modernes. Leur fabrication est réalisée par de nombreuses techniques utilisant, par exemple, la lithographie par faisceau d'électron ou la photolithographie pour graver des photoresists. Grâce à ces procédés, des îlots microscopiques de polymère sont formés et, par recuit, le polymère est ramolii pour créer spontanément la forme sphérique sous l'action des forces de tension superficielle. De manière similaire, avec notre procédé de photoablation $(\mathrm{ArF})$, une matrice îlots cylindriques est produite à partir d'une couche épaisse $(100 \mu \mathrm{m})$ de Téflon AF dopé. La photoablation est réalisée en projetant limage d'un masque (chrome sur quartz) sur l'échantillon de Téflon dopé. Le dopant utilisé est une molécule de tris(perfluoroheptyl)-s-triazine (TPFT) qui a une bonne compatibilité avec le polymère fluoré à température ambiante grâce à ses chaînes latérales perfluorées. De plus, il est éliminé complètement du polymère par recuit, à partir de $160^{\circ} \mathrm{C}$. Pour obtenir la sphéricité des microlentilles, le recuit est poussé jusqu'à $300^{\circ} \mathrm{C}$, la température de fusion du Téflon AF. Comme le montre la Fig.2, une bonne sphéricité a été obtenue. Celle-ci a été mesurée précisément par profilométrie (Alphastep 200 et Tencor P1). Le Téflon $\mathrm{AF}$, qui a d'excellentes qualités optiques grâce à sa transparence, son indice de réfraction faible et sa structure amorphe, s'est révélé être un matériaux de choix pour ces microlentilles. Les lentilles ont été utilisées avec le faisceau du laser à excimères à 193 $\mathrm{nm}$, la longueur d'onde la plus difficile pour les optiques. Des microablations ont été réalisées dans des films de MYLAR et ont permis de mesurer les distances focales $(300 \mu$ $\mathrm{m})$. Des lentilles de taille inférieure $(50 \mu \mathrm{m})$ ont été réalisées mais la technique, que nous avons expérimentée, n'a pas encore été poussée à ses limites inférieures.

\section{Les structurations périodiques induites par le faisceau polarisé du laser à excimère}

Depuis deux ans environ, nous avons montré que lirradiation par le faisceau polarisé à bas flux, c'est à dire à des fluences légèrement en dessous du seuil d'ablation, génère une nouvelle classe de structures périodiques, telles que celles de la Figure.3, sur un certain nombre de polymères. Jusqu'ici les études ont été faites principalement à 193 et $248 \mathrm{~nm}$ en accumulant un grand nombre d'impulsions, typiquement plusieurs milliers, d'énergie située entre 3 et $10 \mathrm{~mJ} / \mathrm{cm}^{2}$, sur les polymères les plus courants comme les PET, PBT, PS, PC, PI, PPQ, $(9,10,11,12,13,14)$ etc (voir Fig.1). L'irradiation peut se faire en présence d'air ou bien sous vide pour la plupart des polymères. Mais certains polymères, comme le polystyrène, ne se structurent pas sous vide, ce qui nous a révélé que l'oxygène est nécessaire dans ce cas. Les expériences ont montré que l'on est en présence d'un nouveau type de structuration de caractère photochimique où il n'y a pas de fusion nette de la surface du matériau et où la transformation de surface s'opère par perte de petites molécules (photolyse ou ablation), avec dans certains cas une activation possible par combinaison avec l'oxygène. Ce mécanisme diffère donc des mécanismes thermocapillaires connus par des études antérieures $\left({ }^{15}\right)$, où un bain liquide est formé par chauffage laser, puis une onde capillaire est excité par le faisceau à sa surface. Sur nos 


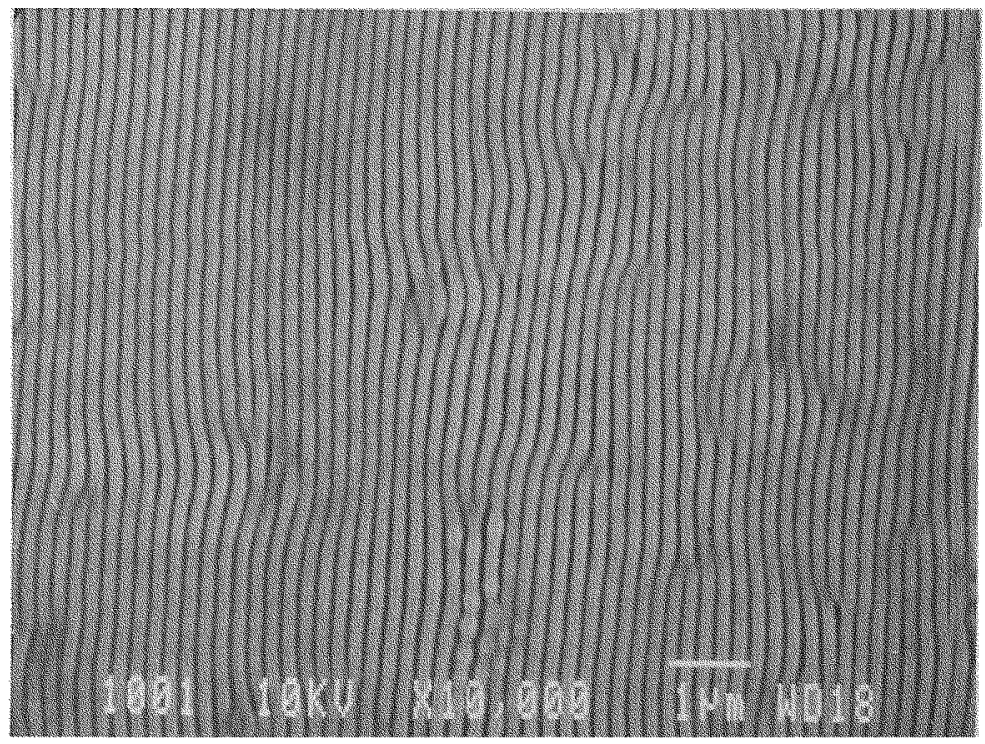

Fig.3 Structures submicroniques obtenues sur le polycarbonate par irradiation avec la radiation polarisée $\mathrm{KrF}$ du laser à excimère dans l'air. Fluence $10 \mathrm{~mJ} / \mathrm{cm}^{2}, 3 \times 10^{3}$ impulsions, incidence normale $\left(\mathrm{i}=0^{\circ}\right.$ ). La direction des structures coïncide avec celle de la polarisation et la période est de $204 \mathrm{~nm}$.

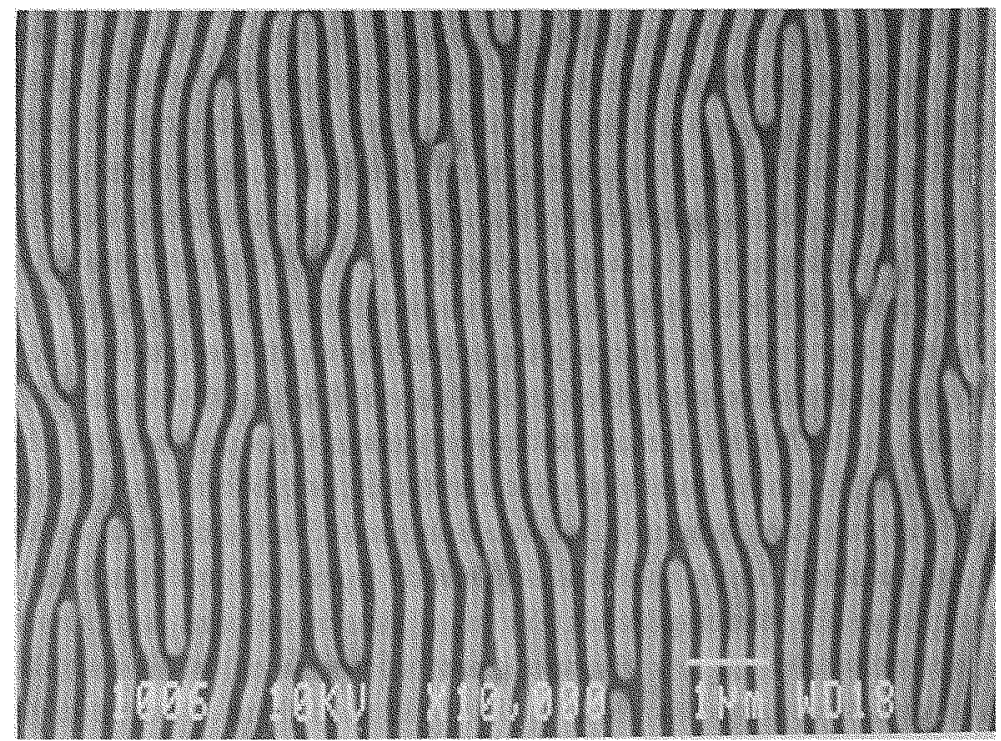

Fig.4 Structures obtenues dans les mêmes conditions que pour la Fig.3 sauf lincidence $\left(\mathrm{i}=30^{\circ}\right.$ ). La période est maintenant agrandie à $362 \mathrm{~nm}$. 
polymères, le taux d'ablation qui est très faible, inférieur à $1 \AA /$ impulsion, n'est pas la contribution dominante à la formation des structures. Il est possible que l'on ait à faire à une migration d'origine thermique de la matière, sous l'effet de forces de tension superficielles, sans qu'un bain de matière fondue soit véritablement formé. Nous savons par des études de surface (ESCA, FTIR) que la surface est fortement modifiée du point de vue chimique, ce qui à pour effet d'augmenter sa sensibilité à l'ablation: c'est le phénomène d'incubation. Il semble plausible que dans certains cas les creux se forment par ablation du matériau incubé, mais jusqu'ici aucune preuve définitive est acquise. Il faut considérer que ces structurations apparaissent à des fluences proches des seuils d'ablation (par exemple à $248 \mathrm{~nm}: 6 \mathrm{~mJ} / \mathrm{cm}^{2}$ pour le PET dont le seuil est $28 \mathrm{~mJ} / \mathrm{cm}^{2}$ ). Il est particulièrement intéressant, pour la détermination des mécanismes comme pour les applications d'identifier les paramètres qui influent sur la période spatiale $\Lambda$ des structures. Nous avons déterminés un certain nombre de ces facteurs, parmi lesquels l'angle d'incidence $\theta$ du faisceau joue le rôle le plus important. Les autres facteurs identifiés, dont dépend beaucoup plus faiblement $\Lambda$, sont la nature du polymère et la fluence. La dépendance en $\theta$ est bien modélisée par l'équation (1) où $\lambda$ est la longueur d'onde du laser et $n$ l'indice de la surface du matériau.

$$
\Lambda=\frac{\lambda}{n \pm \sin \theta}
$$

Ce modèle s'inspire de théories précédentes ${ }^{16}$ faisant intervenir l'existence d'une radiation de surface (se propageant dans la couche d'indice $n$ ), qui, superposée à l'onde incidente résulterait en une modulation périodique de lintensité au voisinage de la surface. L'effet est donc maximum lorsque le faisceau incident est polarisé linéairement, et le modèle prévoit bien que seule la polarisation de type $S$ (ou TE) conduit à une variation de $\Lambda$ avec $\theta$.

L'étude de la cinétique de l'apparition des structures est particulièrement importante car elle met en évidence quatre phases distinctes, qui sont successivement l'incubation (pas de structures), la croissance, la maturité et la décroissance. On observe donc que les structures passent par un maximum, qui est la phase de maturité, puis disparaissent pour donner transitoirement des structures plus grossières et désordonnées, où bien des matrices de points comme celles visibles sur la Figure.5. La disparition peut aller jusqu'au lissage complet de la surface, mais ce comportement n'a pas encore été observé systématiquement. Il semble que la séquence cinétique, incubation-croissance-maturitédécroissance, soit très dépendante de la fluence utilisée. C'est pour cette raison que des structures de caractéristiques différentes peuvent coexister au cours d'une même irradiation, à cause des inhomogénéités d'intensité dans le faisceau laser. Il est possible d'observer des domaines, où les structures sont en croissance, avoisinant d'autres, où elles sont en disparition, car soumis à une intensité plus forte. Comme le phénomène montre un caractère non-linéaire important, de petites fluctuations d'intensité dans le faisceau peuvent s'accompagner d'inhomogénéités de structuration très évidentes. La compréhension de ce problème passe donc par la maîtrise totale de l'homogénéité du faisceau du lașer à excimère. 


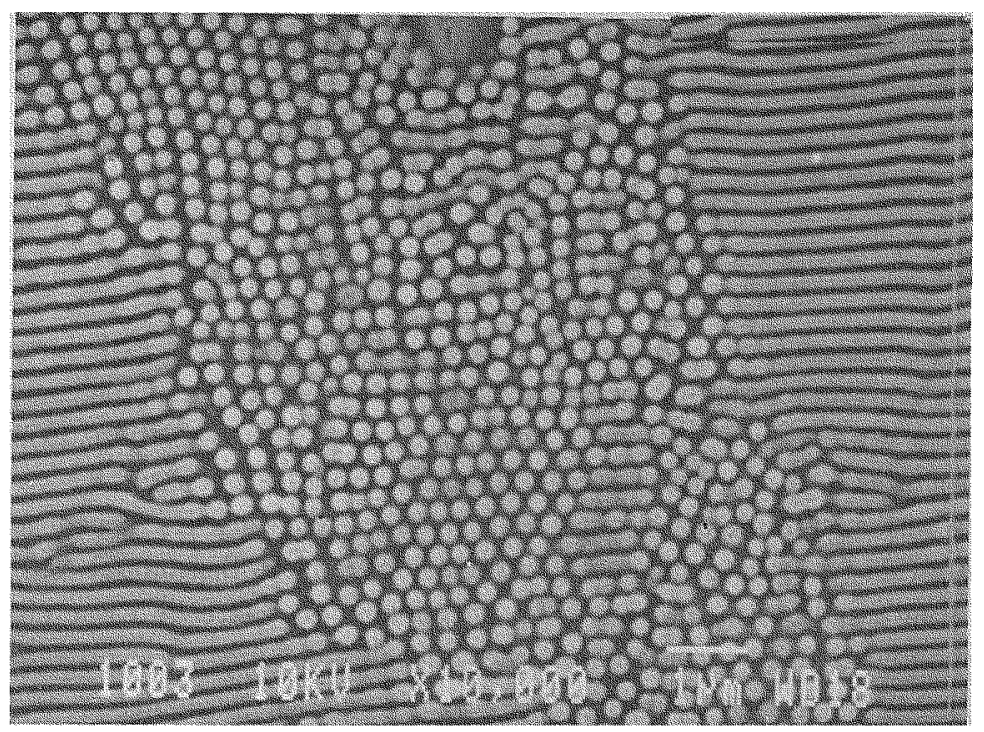

Fig.5 Structuration en points obtenue par irradiation à $248 \mathrm{~nm}$ du polycarbonate. Fluence $10 \mathrm{~mJ} / \mathrm{cm}^{2}, 3 \times 10^{3}$ impulsions, incidence normale $\left(i=0^{\circ}\right)$. Les lignes qui se forment en premier, se fondent ensuite en des points par irradiation prolongée.

\section{Applications des structurations périodiques submicroniques par laser UV}

Nous avons donc découvert un procédé photochimique qui permet très efficacement de structurer des surfaces de polymère à une échelle submicronique. Les limites de la méthode ne sont pas encore connues, mais son importance est déjà bien comprise. Cette approche se présente comme une technique de structuration pour des grandes surfaces, puisqu'il suffit de déplacer le faisceau laser, à la manière d'un pinceau, pour couvrir la surface. L'échelle de rugosité de nos échantillons est accessible par d'autres techniques comme la microlithographie, ou la gravure du type pointe de diamant, utilisée, par exemple pour la fabrication des réseaux. Cependant le progrès, en productivité et simplification de notre méthode, est considéré comme important, puisqu'elle s'adresse à des polymères variés et ordinaires, et est capable de traiter des surfaces comme une feuille de format A4 en l'espace de quelques minutes. De plus, notre méthode offre un contrôle très précis sur les dimensions des structures obtenues, aussi bien la période, qui peut être ajustée par la longueur d'onde et l'incidence, que la hauteur, modulable par le nombre d'impulsions utilisées. Il est possible recenser un certains nombre de propriétés de surface susceptibles d'être améliorées par un tel traitement. II s'agit de la friction $\left({ }^{17}\right.$ ), du mouillage, de l'alignement des cristaux liquides, de l'adhésion, de l'épitaxie, des propriétés optiques, des propriétés aérodynamiques et hydrodynamiques, de l'adsorption et du piégeage de particules. Il est certain que maintenant nous avons un moyen très efficace de produire ces surfaces structurées au laboratoire pour en étudier les propriétés. Les lasers UV modernes affichent des 
puissances considérables, de l'ordre du $\mathrm{kW}$, qui pourront satisfaire des besoins industriels, avec une vitesse de traitement de l'ordre du mètre carré par minute. Notons encore qu'une technique photochimique telle que celle-ci est aussi compatible avec une technologie utilisant le vide.

\section{Conclusions}

La photoablation des polymères a rapidement conduit les expérimentateurs à découvrir de nouvelles structures périodiques de surface, de taille submicronique et qui apparaissent à des énergies légèrement en dessous des seuils d'ablation. Comme pour d'autres structures déjà connues, l'intensité laser est modulée par interférence avec une radiation de surface diffusée par la rugosité du polymère. Ensuite, les mécanismes mis en jeu sont plutôt du type photochimique (par opposition à photothermique) oủ des petites molécules sont expulsées du polymère. Le phénomène peut être de caractère ablatif à cause des modifications de surface importantes qui sont impliquées. Les premières études ont montré un potentiel important d'applications.

[1] R.Srinivasan, B.Braren, Chem.Rev., 89, 1303 (1989); S.Lazare et V.Granier, Laser Chem., 10, 25 (1989).

[2] M.Bolle, S.Lazare, M.Leblanc, A.Wilmes, Appl.Phys.Lett. 60, 674 (1992)

[3] M.Bolle, S.Lazare, J.Appl.Phys. 73, 3516 (1993)

[4] S.Lazare, Techniques d'utilisation des photons: Principes et applications, Collection ELECTRA Editions DOPEE85, 447 (1992), Chapitre 7.1

[5] F. Bachmann, Chemtronics, , 4, 149 (1989).

[6] J.R.Lankard, G.Wolbold, Appl.Phys. A, 54, 355 (1992)

[7] H.Hiraoka et S.Lazare, Appl.Surf.Sci., 46, 342 (1990).

[8] S.Mihailov et S.Lazare, Appl.Opt., 32, 6211 (1993).

[9] M.Bolle et S.Lazare, Appl.Surf.Sci., 69, 31 (1993)

[10] M.Bolle et S.Lazare, Appl.Surf.Sci., 65/66, 349 (1993)

[11] S.Lazare, P.Benet, M.Bolle, P.De Donato, E. Bernardy, SPIE Gas Flow and Chem.Lasers, 1810, 546 (1992)

[12] M.Bolle et S.Lazare, AIP Conf.Proc. Laser Ablation: Mechanisms and Applications- $I I, 288,550$ (1994)

[13] M.Bolle, S.Lazare, D.Drilhole, Polyimides and Other High Temperature Polymers, M.Abadie and S.Sillon Editors, sous presse, (1994)

[14] M.Bolle, thèse, Université de Bordeaux I, juin 1994.

[15] voir revue de la littérature dans la référence 3, et plus récemment V.N.Tokarev et V.I.Konov, J.Appl.Phys., 76, 800 (1994).

[16] A.E.Siegman, P.M. Fauchet, IEEE J.Quantum Electron., QE-22, 1384 (1986).

[17] G.Fisher, A.Hagemeyer, H.Hibst, H.J.Richter, H.P.Schilberg, S.Lazare, M.Bolle, Brevet BASF Magnetics Gmbh déposé le 31 juil. 92 sous le $n^{\circ}$ d'enregistrement allemand No P 4225554.6 O.Z. 0078/06073 